\title{
The use of spirometry in a primary care setting
}

This article was published in the following Dove Press journal:

International Journal of General Medicine

30 September 2009

Number of times this article has been viewed

\section{Elizabeth A Blain \\ Timothy J Craig}

Penn State Hershey Medical Center, Hershey, PA, USA
Correspondence: Elizabeth A Blain Penn State Hershey Medical Center, 500 University, Box H039, Hershey,

PA 17033, USA

Tel $+|7| 753 \mid 8390$

Email eblain@hmc.psu.edu
Objective: To determine the use of spirometry in family practice, internal medicine, and pediatric outpatient settings.

Methods: Data were collected from 45 outpatient offices in the central Pennsylvania area via phone survey that asked a set of four questions: 1) Do you have spirometry in your office? 2) Do you use spirometry for asthma patients? 3) In what situation do you use spirometry for? 4) Do you use spirometry more for chronic obstructive pulmonary disease (COPD) or asthma? Results: It was found that pediatricians used spirometry $66 \%$ of the time, family practitioners $47 \%$ of the time, and internal medicine practitioners $60 \%$ of the time. Of those who did not use spirometry, $94 \%$ stated that they refer to a hospital if they required spirometry and $6 \%$ referred to subspecialists if the patient required spirometry. $10 \%$ of pediatricians performed the test on each asthma visit, otherwise the others used it only for exacerbations or as a baseline. No internists used spirometry regularly for asthma patients, and $22 \%$ used it more for COPD. In family practice only $14 \%$ used spirometry routinely at each visit for asthma patients.

Conclusions: Pediatricians used spirometry more often in the outpatient setting than other specialists, followed closely by internal medicine physicians. Family practice physicians were the least likely to use spirometry. Multiple barriers seemed to prevent routine use of spirometry, but no one barrier accounted for the majority.

Keywords: spirometry, asthma, primary care

\section{Introduction}

Spirometry has been an important part in asthma diagnosis, assessing risk of future adverse events, and guiding treatment because it is one of the few objective parameters used in asthma that can change the assessment and treatment of asthma. ${ }^{1}$

"The expert panel recommends the following frequencies for spirometry measurements: at the time of initial assessment; after treatment is initiated and symptoms and PEF have stabilized, to document attainment of (near) "normal" airway function; during a period of progressive or prolonged loss of asthma control; and at least every 1-2 years to assess the maintenance of airway function. Spirometry may be indicated more often than every 1-2 years, depending on the clinical severity and response to management. These spirometry measures should be followed over the patient's lifetime to detect potential for decline and rate of decline of pulmonary function over time."'

The use of spirometry could help detect cases at an early stage when intervention may prevent further progression of the disease. ${ }^{2}$ Spirometry is also important because studies have shown poor correlation between subjective symptoms and 
the degree of obstruction on spirometry, and in one study, up to $27 \%$ of physicians using symptoms alone for diagnosis underestimated asthma severity. ${ }^{3}$

A national survey eight years ago found that only a fifth of primary care providers used spirometry on a regular basis. ${ }^{4}$ In one study, asthma specialists were more likely to have an office spirometer and use it than were primary care physicians. ${ }^{5}$ In the same study, primary care physicians were more likely to use a spirometer if they agreed the data was necessary for accurate diagnosis and care, and if they believed they could be appropriately trained to perform and interpret the test correctly. ${ }^{5}$ This study was conducted to assess the use of spirometry care in primary care, based on speciality, in order to help direct where education on this technique should be directed and to see if there is greater utilization of spirometry compared to almost a decade ago.

\section{Methods}

Sixty-nine primary care offices in central Pennsylvania were contacted by phone in 2008 to determine if they utilized spirometry and to assess barriers for the use of spirometry. Spirometry was differentiated from the use of peak expiratory flow meters. The survey was conducted by questioning the office manager of the practice. Forty-five offices answered the survey, 15 in each specialty. They were asked a series of questions as follows: Do you use spirometry in your office? If you do not use spirometry in the office do you refer them for spirometry? If you use a spirometer in your office do you use it more often to assess asthma or chronic obstructive pulmonary disease (COPD)? Do you use spirometry to establish a baseline, for exacerbations, both or at every visit? Questions regarding COPD were omitted in pediatrician offices.

\section{Results}

Pediatricians used spirometry $10 \%$ of the time on patients with asthma at every visit, $30 \%$ of the time to achieve a baseline, $10 \%$ of the time during an exacerbation only, $40 \%$ of the time for both baseline and exacerbation, and $10 \%$ of the time for reasons other than asthma, which they did not specify. None of the internal medicine physicians used spirometry at every asthma visit, but did use it $33 \%$ of the time to establish a baseline, $44 \%$ of the time during an exacerbation, $11 \% \mathrm{did}$ not use it for asthma at all, and 11\% owned a spirometer, but never used it. $22 \%$ of internists used spirometry more for COPD than asthma. Family medicine physicians used spirometry $14 \%$ of the time at every visit, $29 \%$ of the time for baseline, $29 \%$ of the time for exacerbations only, and $29 \%$ for both exacerbations and baselines. No family medicine physician used it for a reason other than asthma (Figure 1). Pediatricians use spirometry the most at $66 \%$, followed by internal medicine at $60 \%$, and family medicine last at $47 \%$ (Figure 2). Of those who did not use spirometry, 94\% of these physicians referred to a hospital if they needed one, and $6 \%$ referred to a subspecialist.

\section{Discussion}

Primary care physicians provide care to about $66 \%$ of asthmatics in the US, but seem to underutilize spirometry

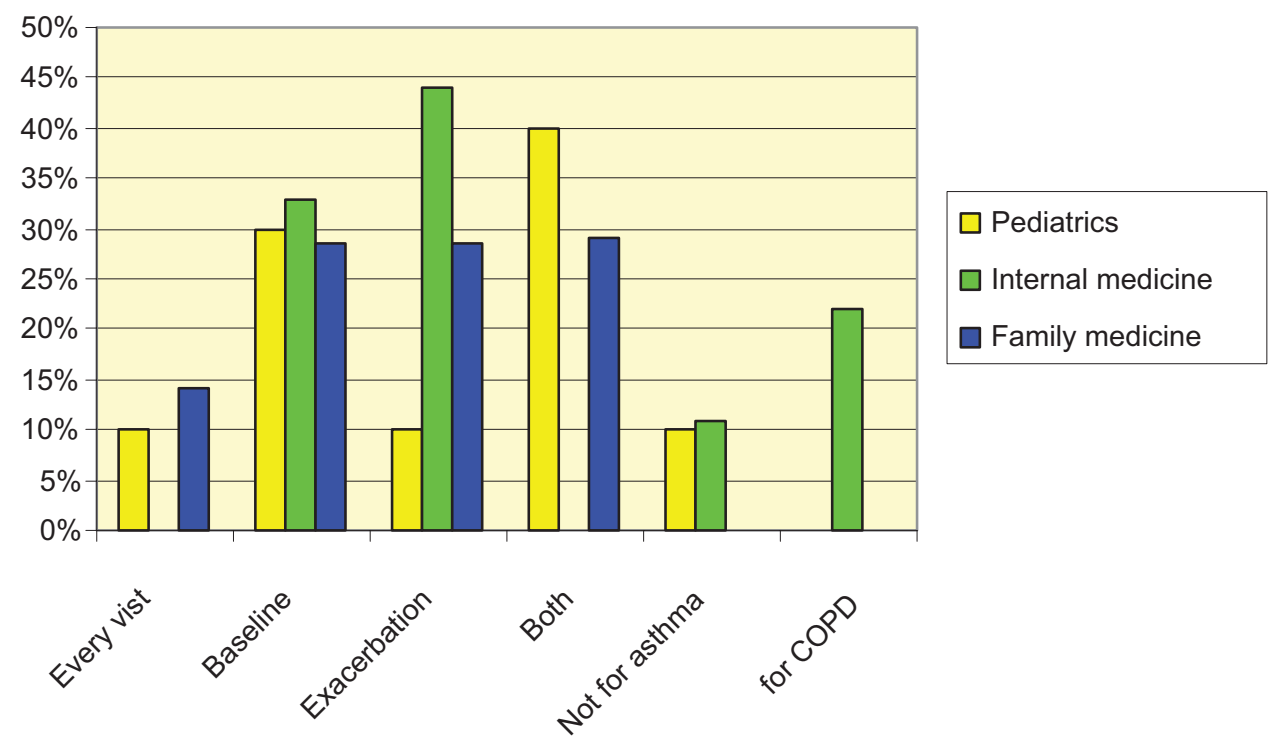

Figure I Reasons for the use of spirometry in primary care based on specialty. Abbreviation: COPD, chronic obstructive pulmonary disease. 


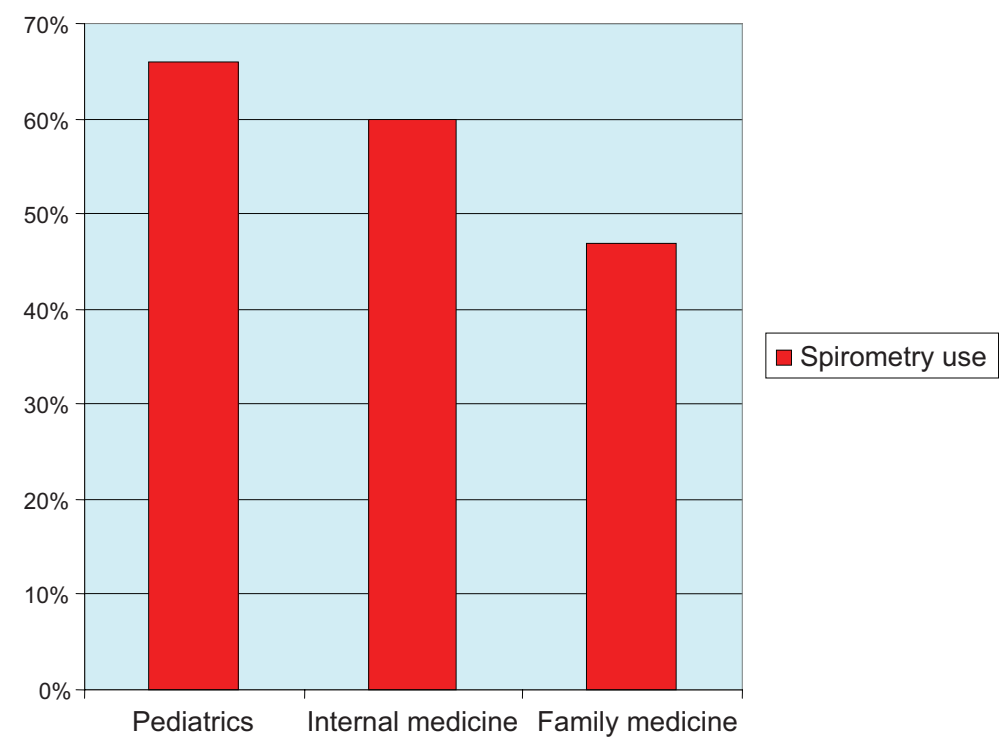

Figure 2 Percent usage of spirometry in the subspecialties of primary care.

as a tool to diagnose and serially follow the course of asthma. Despite recommendations about optimal care for patients with asthma by the National Asthma Education and Prevention Program, spirometry is still not being used to assess the majority of patients with asthma. Our data suggest that pediatricians utilize spirometry to the greatest extent, and this is likely secondary to the past efforts to teach pediatricians about spirometry, how to utilize spirometry to optimize care of patients with asthma and how to interpret the results. ${ }^{4,6-12}$ While the usage of spirometry still seems low, our study shows increased frequency of its use over what has been demonstrated previously. This suggests that the NHLBI Guidelines for Management of Asthma have influenced care for patients with asthma. The increase utilization of inhaled corticosteroids and decreasing mortality associated with asthma are other factors that seem to suggest that guidelines do have an impact on asthma care.

Studies in the past have shown that interpretation of spirometry by family physicians following training was almost equivalent to that of pulmonologists. ${ }^{6}$ Appropriate interpretation also impacted the change in therapy in as much as $30 \%$ of patients. When the treatment was changed the provider increased therapy approximately $75 \%$ of the time. ${ }^{6}$ This data suggests that that the use of spirometry may improve asthma outcomes, and that utilization of this technique should increase. This is especially true for family medicine physicians who seem to be utilizing it the least.

This study's main limitation is limited sample size. We also failed to elicit the barriers that prevented the use of in office spirometry. In addition, we did not query as to what prompted an outside referral for spirometry. We suspect that one barrier to its use is that there is a concern about proper interpretation. Another potential barrier is the concern that adequate technique will limit the benefit of spirometry. This latter concern is somewhat supported by prior literature that suggests spirometry results are more accurate when the technician performing them has more experience. A study in Spain showed that a mere $48 \%$ of spirometry tests were considered acceptable. ${ }^{13}$ Nonetheless, newer spirometers, with user friendly software and patient friendly soft ware, improve the results even when the spirometer is not being used multiple times a day.

It appears that education on spirometry would enhance its utilization in primary care if it focused on how to purchase and maintain a spirometer, perform spirometry, interpret the results, correctly invoice for the services, and how the results can be used to improve asthma management. There also appears to be an opportunity for allergists to perform spirometry in their offices as a service for primary care physicians. This may decrease cost and improve availability both which may increase utilization of the procedure by primary care physicians.

\section{Disclosures}

The authors report no conflicts of interest in this work.

\section{References}

1. National Asthma Education and Prevention Program. Expert Panel Report 3 (EPR-3): Guidelines for the Diagnosis and Management of Asthma-Summary Report 2007. J Allergy Clin Immunol. 2007; 120(5 Suppl):S94-S138.

2. Celli BR. The importance of spirometry in COPD and asthma. Chest. 2000;117:15S-19S. 
3. Yawn BP, Enright PL, Lemanske RF Jr, et al. Spirometry can be done in family physicians' offices and alters clinical decisions in management of asthma and COPD. Chest. 2007;132(4):1162-1168.

4. Finkelstein JA, Lozano P, Shulruff R, et al. Self-reported physician practices for children with asthma: are national guidelines followed? Pediatrics. 2000;106:886-896.

5. O'Dowd LC, Fife D, Tenhave T, Panettieri RA Jr. Attitudes of physicians toward objective measures of airway function in asthma. Am J Med. 2003;114(5):391-396.

6. Holt EW, Tan J, Hosgood HD. The impact of spirometry on pediatric asthma diagnosis and treatment. J Asthma. 2006;43(7):489-493.

7. Cabana M, Slish KK, Nan B, Leo H, Bratton SL, Dombkowski KJ. Outcomes associated with spirometry for pediatric asthma in a managed care organization. Pediatrics. 2006;118(1):e151-e156.

8. Nair SJ, Daigle KL, DeCuir P, Lapin CD, Schramm CM. The influence of pulmonary function testing on the management of asthma in children. Pediatrics. 2005;147(6):797-801.

9. Zanconato S, Meneghelli G, Braga R, Zacchello F, Baraldi E. Office spirometry in primary care pediatrics: a pilot study. Pediatrics. 2005; 116(6):e792-e797.

10. Poels PJ, Schermer TR, Jacobs A, et al. Variation in spirometry utilization between trained general practitioners in practices equipped with a spirometer. Scand J Prim Health Care. 2006;24(2):81-87.
11. Lucas AE, Smeenk FW, Smeele IJ, van Schayck CP. Overtreatment with inhaled corticosteroids and diagnostic problems in primary care patients, an exploratory study. Fam Pract. 2008;25(2):86-91.

12. Grant EN, Moy JN, Turner-Roan K, Daugherty SR, Weiss KB. Asthma care practices, perceptions, and beliefs of Chicago-area primary-care physicians. Chicago Asthma Surveillance Initiative Project Team. Chest. 1999;116:145S-154S.

13. Martínez Eizaguirre JM, Irizar Aranburu MI, Estirado Vera C, Berraondo Zabalegui I, San Vicente Blanco R, Aguirre Canflanca E. Quality of spirometry tests done in primary care units in the province of Gipuzkoa. Aten Primaria. 2008 May;40(5):235-259.

14. Shim CS, Williams MH Jr. Evaluation of the severity of asthma: patients versus physicians. Am J Med. 1980;68:11-13.

15. Tsuyuki RT, Sin DD, Sharpe HM, Cowie RL, Nilsson C, Man SF; Alberta Strategy to Help Manage Asthma (ASTHMA) Investigators. Management of asthma among community-based primary care physicians. J Asthma. 2005;42(3):163-167.

16. Gharagozlou M, Abdollahpour H, Moinfar Z, Bemanian MH, Sedaghat M. A survey of pediatricians' knowledge on asthma management in children. Iran J Allergy Asthma Immunol. 2008;7(2):85-90.

17. Cowen MK, Wakefield DB, Cloutier MM. Classifying asthma severity: objective versus subjective measures. J Asthma. 2007;44(9): 711-715.

\section{Publish your work in this journal}

The International Journal of General Medicine is an international, peer-reviewed open-access journal that focuses on general and internal medicine, pathogenesis, epidemiology, diagnosis, monitoring and treatment protocols. The journal is characterized by the rapid reporting of reviews, original research and clinical studies across all disease areas.

\section{Dovepress}

A key focus is the elucidation of disease processes and management protocols resulting in improved outcomes for the patient. The manuscript management system is completely online and includes a very quick and fair peer-review system. Visit http://www.dovepress.com/ testimonials.php to read real quotes from published authors. 